\title{
Fe I Line Asymmetries and Shifts Caused by Pressure Broadening
}

\author{
V. Kršljanin, I. Vince and S. Erkapić \\ Astronomical Observatory, Volgina 7, 11050 Belgrade, Yugoslavia
}

\begin{abstract}
In order to test the validity of the usual neglect of pressure line shifts in investigations of $\mathrm{Fe} I$ line asymmetries and shifts, we calculated synthetic line bisectors and shifts of 96 moderate and weak photospheric lines, taking into account both hydrogenand electron-impact shifts and neglecting the velocity fields. Absolute pressure line shifts obtained are less than or appr. equal to $50 \mathrm{~m} / \mathrm{s}$ and the largest relative shifts along the bisectors are about $40 \mathrm{~m} / \mathrm{s}$. We found pronounced dependences of both shifts and bisectors on the energy of the upper level in transitions and on the transition array.
\end{abstract}

Pressure broadening produces small (Vince et al., 1985b), but some cases observable (Vince and Dimitrijević, 1989) line asymmetries and shifts of $\mathrm{NaI}$ lines. It was shown recently (Kršljanin, 1989) that some solar Fe I lines also exhibit measurable pressure broadening effects. Estimates of Fe I lines hydrogen-impact shifts (Gomez et al., 1987) appear to be insufficient to determine overall pressure shifts, since collisions with electrically charged particles can produce line shifts of the same order of magnitude as collisions with hydrogen atoms do (Vince et al., 1985a,b). On the other hand, detailed theoretical calculations of $\mathrm{Fe} I$ lines pressure widths and particularly shifts are still missing due to complicated atomic structure of iron.

In order to estimate the importance of pressure broadening contribution to the observed asymmetries and shifts of Fe I lines we therefore decided to investigate averaged pressure broadening effects on many Fe I lines using simple theoretical approaches to hydrogen-impact (Hindmarsh et al., 1967) and electron-impact shifts (Kršljanin, 1990). These approaches give good average accuracy when applied to many lines. So, such a method promise to give results comparable with previous convective motion investigations (e.g. Dravins et al., 1981) performed also on large samples of Fe I lines.

We synthetisied, using a LTE code and Holweger-Müller (1974) photospheric model, profiles of $96 \mathrm{Fe}$ I lines (from the list of Dravins et al., 1981) in the centre of the solar disk, with hydrogen- and electron-impact shifts taken into account as the only causes of wavelength displacements in the line profiles. Lines were chosen that had observed central line depths less or equal to 0.5 and for which reliable atomic data was available ( $g f$ values at least of accuracy C according to Fuhr et al., 1981). Wavelength shifts and averaged bisectors (normalised to central intensities) of the synthetic line profiles are presented in Fig. 1. The bisector shapes are mainly determined by perturbed density distribution in the line formation regions and often by opposite sings of hydrogen- and electron-impact shifts. The shifts of the line bottoms and the relative wavelength displacements along the bisectors are 

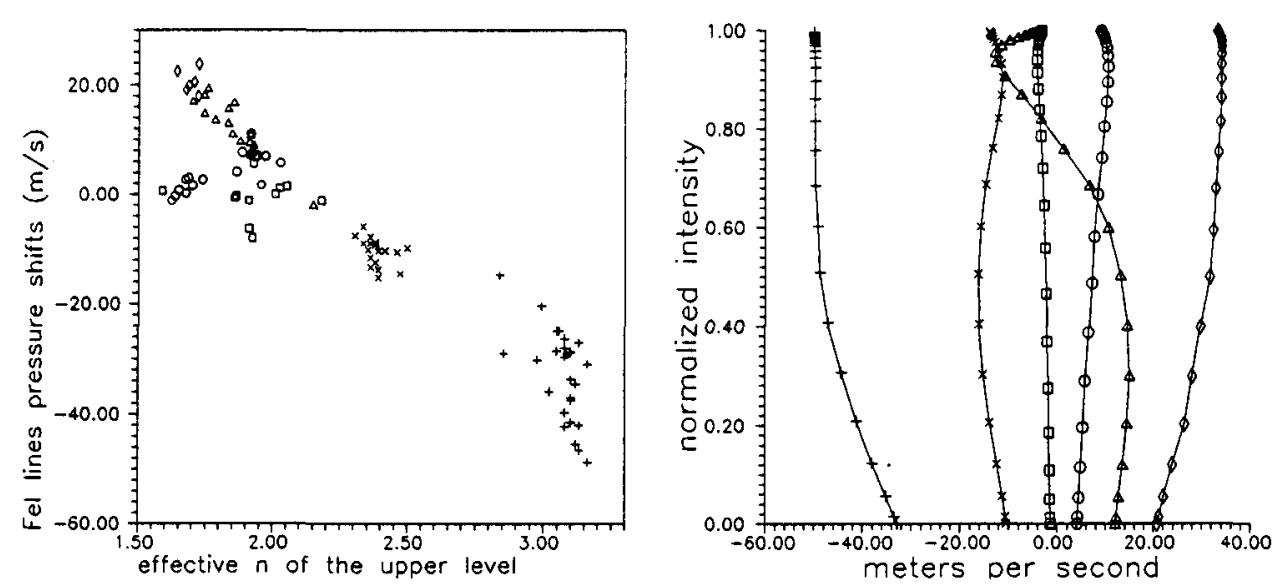

Fig. 1. Fe I lines pressure shifts (left) and averaged bisectors (right) for the transition arrays: $(\Delta) 3 d^{8}-4 p ;(\sqcap) 4 s^{2}-4 p$ with lower energy of the upper level parent terms; $(\diamond) 4 s^{2}-4 p ;(\bigcirc) 4 s-4 p ;(\times) 4 p-5 s ;(+) 4 p-4 d$.

very small in comparison with the observed ones (Dravins et al., 1981). Another important fact which appears from our results is the pronounced dependence of the pressure shifts and line bisectors on the energy of the upper level in the transitions, i.e. on the effective principal quantum number of the upper level, and on the type of transition, i.e. on the transition array. Such a dependence, if eventually found in stellar observational data, might be an indicator of an important pressure broadening contribution to the observed line shapes. However, even in the solar case the pressure shifts should be considered for some Fe I lines (e.g. for lines from the $4 p-4 d$ transition array with relatively large pressure shifts and from the $3 d^{8}-$ $4 p$ transition array with relatively large bisector curvatures). Our calculations give an estimate of the value of pressure broadening effects in diagnostics of convective motions from the shifts and shapes of Fe I lines.

\section{References}

Dravins, D., Lindgren, L., Nordlund, Å.: 1981, Astron. Astrophys. 96, 345

Fuhr, J.R., Martin, G.A., Wiese, W.L., Younger, S.M.: 1981, J. Phys. Chem. Ref. Data 10, 305

Gomez, M.T., Marmolimo, C., Roberti, G., Severino, G.: 1987, Solar Phys. 112, 227

Hindmarsh, W.R., Petford, A.D., Smith, G.: 1967, Proc. Roy. Soc. A297, 296

Holweger, H., Müller, E.A.: 1974, Solar Phys. 39, 19

Kršljanin, V.: 1989, in Solar and Stellar Granulation, eds. R.J. Rutten and G. Severino, Kluwer, Dordrecht, p. 91

Kršljanin, V.: 1990, in 22nd EGAS, ed. A. Wännström, Dept. Phys. Univ. Uppsala, p. 757

Vince, I., Dimitrijević, M.S.: 1989, in Solar and Stellar Granulation, eds. R.J. Rutten and G. Severino, Kluwer, Dordrecht, p. 93

Vince, I., Dimitrijević, M.S., Kršljanin, V.: 1985a, in Spectral Line Shapes, ed. F. Rostas, W. de Gruyter, Berlin, p. 649

Vince, I., Dimitrijević, M.S., Kršljanin, V.: 1985b, in Progress in Stellar Spectral Line Formation Theory, eds. J.E. Beckman and L. Crivellari, D. Reidel, Dordrecht, p. 373 